\title{
COUPLING BETWEEN MICROSTRIP LINES EMBEDDED IN POLYIMIDE LAYERS FOR 3D-MMICs ON Si
}

\author{
George E. Ponchak ${ }^{1}$, Emmanouil M. Tentzeris ${ }^{2}$, and John Papapolymerou ${ }^{2}$ \\ 1. NASA Glenn Research Center, 21000 Brookpark Rd., MS 54/5, Cleveland, OH 44135 USA \\ 2. School of Electrical and Computer Engineering, Georgia Institute of Technology, Atlanta, GA 30332-0250
}

Abstract - Three-dimensional circuits built upon multiple layers of polyimide are required for constructing Si/SiGe monolithic microwave/millimeter-wave integrated circuits on CMOS (low resistivity) Si wafers. However, the closely spaced transmission lines are susceptible to high levels of coupling, which degrades circuit performance. In this paper, Finite Difference Time Domain (FDTD) analysis and measured characteristics of novel shielding structures that significantly reduce coupling between embedded microstrip lines are presented. A discussion of the electric and magnetic field distributions for the coupled microstrip lines is presented to provide a physical rationale for the presented results.

Keywords: microstrip, coupling, crosstalk

\section{INTRODUCTION}

There is a rapidly expanding market for Si Microwave/Millimeter-Wave Integrated Circuits (MMICs) fabricated in standard CMOS foundries to replace GaAs MMICs in wireless communication systems, phased array radar, and other applications where the circuit cost is a major factor in determining the system cost. However, microwave passive elements and transmission lines placed directly on standard CMOS grade Si have low quality factors (high attenuation), which necessitates novel transmission line structures [1] that are typically embedded in polyimide that is deposited over 
the Si substrate. Moreover, highly integrated systems that include the RF circuits, digital data processing circuits, sensor circuits, and bias control circuits on a single chip or within a single package also rely on multiple layers of polyimide to construct three-dimensional circuits that are smaller than what would normally be possible.

Although thin film microstrip (TFMS) embedded in polyimide solves the problem of high attenuation and smaller sized circuits, closely spaced transmission lines also increases the potential for high levels of coupling between lines. If the interline crosstalk is too high, the circuit characteristics are severely degraded. Thus, techniques and layout rules are required to reduce coupling between parallel TFMS lines. Prior papers on reducing coupling between microstrip lines built on Low Temperature Cofired Ceramic (LTCC) have shown that a roll of via holes placed between the two lines reduces coupling by $8 \mathrm{~dB}$ if the via holes are connected on the top and bottom by a strip and the ground plane respectively [2,3]. A continuous metal filled wall fabricated between two TFMS lines embedded within polyimide has been shown to also reduce coupling by approximately $8 \mathrm{~dB}$ for a single microstrip geometry [4], and preliminary work by the authors has shown that metal filled via post fences in polyimide provides the same level of coupling reduction [5].

In this paper, a systematic evaluation of the coupling between TFMS lines embedded in polyimide built upon CMOS grade Si is presented for the first time. This in depth characterization includes coupling between microstrip lines on the same polyimide layer and between microstrip lines on different layers of polyimide layers. In addition, the use of metal filled via hole fences and metal walls embedded in the polyimide to reduce coupling is investigated. Finite Difference Time Domain (FDTD) analysis and measurements are used to quantify the coupling, and FDTD generated electric and magnetic field plots are used to qualitatively describe the nature of the coupling.

\section{MiCROSTRIP CIRCUIT DESCRIPTION}

Figure 1 shows a cross sectional cut through microstrip lines embedded in polyimide upon a Si substrate. The microstrip ground plane covers the Si substrate, which shields all of the electromagnetic fields from the lossy Si. W1 and W2 are $23 \mu \mathrm{m}$ and $52 \mu \mathrm{m}$ respectively to yield a $50 \Omega$ transmission line for the polyimide thickness, h, of $10 \mu \mathrm{m}$. Several shielding structures between the two microstrip lines are characterized. For microstrip lines on the same polyimide layer as shown in Figure 1a, the shielding structures are: a roll of metal filled via holes through polyimide layer 1 only, a roll of metal filled via holes through both layers of polyimide, and a continuous, metal filled trench through both layers of polyimide. For microstrip lines on different layers of polyimide as shown in Figure 1b, the shielding structures are a roll 
of metal filled via holes through both layers of polyimide and a metal filled trench through both layers of polyimide. In all cases, the via holes are 20 by $20 \mu \mathrm{m}$ and circuits are analyzed with the via hole spacing, DV, of 60 and $100 \mu \mathrm{m}$ from center to center. Furthermore, all via holes are connected by a continuous, $20 \mu \mathrm{m}$ wide metal strip on each layer as recommended in [2]. The metal filled trench is $20 \mu \mathrm{m}$ wide.

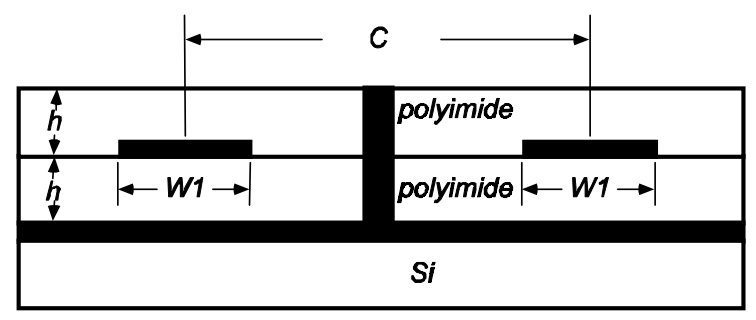

(a)

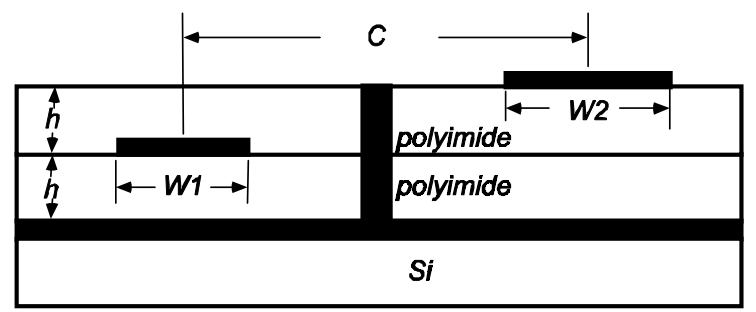

(b)

Figure 1: Cross sectional cuts through microstrip lines embedded in polyimide layers with a shielding structure between them. (a) microstrip lines on same layer of polyimide and (b) microstrip lines on different layers of polyimide.

\section{THEORETICAL ANALYSIS}

The full-wave FDTD technique [6] is used for the theoretical characterization of the forward and backward coupling, $\mathrm{S}_{31}$ and $\mathrm{S}_{41}$ respectively, between the two parallel microstrip lines (see Figure 2). The E- and H-field components are implemented in a leapfrog configuration. An adaptive grid with neighboring cell aspect ratio smaller or equal to 2 maintains a second-order global accuracy.

Numerical meshes of $80-120$ by 45 by 250 cells terminated with 10 Perfectly Matched Layer (PML) cells in each direction provide accurate results for a time-step of $\Delta \mathrm{t}=0.99 \Delta \mathrm{t}_{\max }$. A Gaussian pulse with $\mathrm{f}_{\max }=60 \mathrm{GHz}$ is applied vertically 
as a soft source close to the front end of the microstrip, and its values get superimposed on the FDTD calculated field value for all cells in the excitation region for each time-step. The via holes are modeled as rectangular metal tubes with cross-section $23 \times 20 \mu \mathrm{m}$. To account for the coupling of even and odd modes, two simulations are performed for each geometry exciting both lines with equal amplitude and even or odd space distributions respectively. In addition, both microstrip lines are terminated with matched loads $\left(\mathrm{Z}_{0}=50 \Omega\right)$ that are realized as the combination of shunt resistors placed between the microstrip and the bottom ground [7]. Two probes placed at the front end and at the far end of one line are used for the combination of the results of the even and of the odd simulations. The application of the FFT algorithm derives the frequency-domain results from the time-domain data (usually 25,000 time-steps).

\section{CirCUIT FABRICATION AND EXPERIMENTAL PROCEDURE}

The circuit for experimentally characterizing coupling between the microstrip lines is shown in Figure 2 . The four-port circuit has probe pads orientated so that each port may be probed simultaneously. The coupling region, or the section of parallel transmission lines labeled L in Figure 2, is $5000 \mu \mathrm{m}$ long. Note that for characterization, the shielding structure extends past the transmission line right angle bend and is tapered at 45 degree to minimize the effects of radiation from the right angle bend.

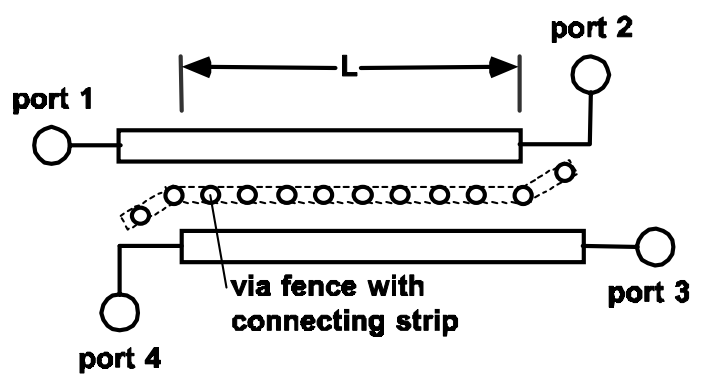

Figure 2: Schematic of the coupled line structures used to characterize the coupling.

The four port microstrip circuits are fabricated on a $1 \Omega$-cm Si wafer. A ground plane consisting of a $300 \AA \mathrm{Ti}$ adhesion layer, $1.5 \mu \mathrm{m}$ of $\mathrm{Au}$, and a $200 \AA \mathrm{Cr}$ cap layer is first evaporated onto the Si wafer. This is followed by spinning on Dupont adhesion promoter and $10 \mu \mathrm{m}$ of Dupont PI-2611 polyimide, which has a permittivity of 3.12 measured at 1 $\mathrm{MHz}$ [8] and a loss tangent of 0.002 measured at $1 \mathrm{kHz}$ [9]. After curing the polyimide at $340 \mathrm{C}$ for 120 minutes, Ni is 
evaporated onto the polyimide to serve as a mask for the $\mathrm{O}_{2} / \mathrm{CF}_{4}$ reactive ion etching (RIE) of the via holes. After the via holes are etched and the Ni removed, $200 \AA$ of Ti and $2000 \AA$ of Au are sputtered onto the wafer to serve as a seed layer for the $1.3 \mu \mathrm{m}$ of Au electroplating that is used to define the embedded microstrip lines and fill the via holes in a single step. These Au microstrip lines are capped with $200 \AA$ of $\mathrm{Cr}$ before applying the next, $10 \mu \mathrm{m}$ layer of polyimide. Thus, all metal structures are $1.5 \mu \mathrm{m}$ thick. This process is repeated for each layer of polyimide. After each step, a DEKTAK surface profile is used to measure the polyimide and metal strip thickness. Both, the DEKTAK and SEM analysis show that the surface roughness is low enough that it can be neglected in the analysis.

Measurements are made on a vector network analyzer from 2 to $50 \mathrm{GHz}$. A Thru/Reflect/Line (TRL) calibration is implemented with MULTICAL [10], a TRL software program, using four delay lines of 1800, 2400, 4800, and $10000 \mu \mathrm{m}$ and a short circuit reflect fabricated on the same substrate as the circuits. To improve accuracy, each circuit is measured several times and the average of those measurements is presented in this paper. During the measurement of the four-port circuits, two of the four ports are terminated in $50 \Omega$ loads built into specially designed picoprobes.

\section{MicRostrip COUPLING RESUlTS}

As a first step, the measured and FDTD analysis results for the embedded microstrip lines are compared across the entire frequency band of 2 to $50 \mathrm{GHz}$. One such case for coupled microstrip lines without any shielding structure is shown in Figure 3. It is seen that there is excellent agreement between the theory and the measured results, which is typical of the other cases. Also typical of all of the results presented in this paper, the forward coupling increases monotonically with frequency, while the backward coupling is periodic. Thus, throughout the paper, the backward coupling results presented are the maximum coupling value over the frequency band. Presented results are backward coupling defined as $-20 * \log \left|\mathrm{S}_{41}\right|$ and forward coupling defined as $-20 * \log \left|\mathrm{S}_{31}\right|$. Note that the definition for forward coupling presented here differs from the definition typically used in the literature which is $S_{31} / S_{21}$ [11], but if the coupling and attenuation for the lines is very small, $\left|\mathrm{S}_{21}\right|$ is approximately equal to one and the two definitions are equivalent. Also, backward coupling is often called near end coupling and forward coupling is often called far end coupling in the literature [11].

While all of the results in this paper are presented for two coupled lines with a coupling length of $5000 \mu \mathrm{m}$, an FDTD analysis of microstrip lines with shielding structures as a function of coupling length was performed. Figure 4 shows that 
the S-parameters for two microstrip lines separated by $\mathrm{C}=72 \mu \mathrm{m}$ and a continuous metal filled trench. As seen in Figure 4a, the maximum level of backward coupling is independent of the coupling length and only the periodicity of the nulls changes. The magnitude of the forward coupling for shielded microstrip lines with different coupling lengths varies with frequency in a similar manner as shown in Figure 3, which is for unshielded coupled microstrip lines, and to a first order, the forward coupling increases linearly with coupling length. These results agree with the general conclusions for coupling between weakly coupled transmission lines [11], which shows that the shielding structures do not change the basic physics that cause coupling.

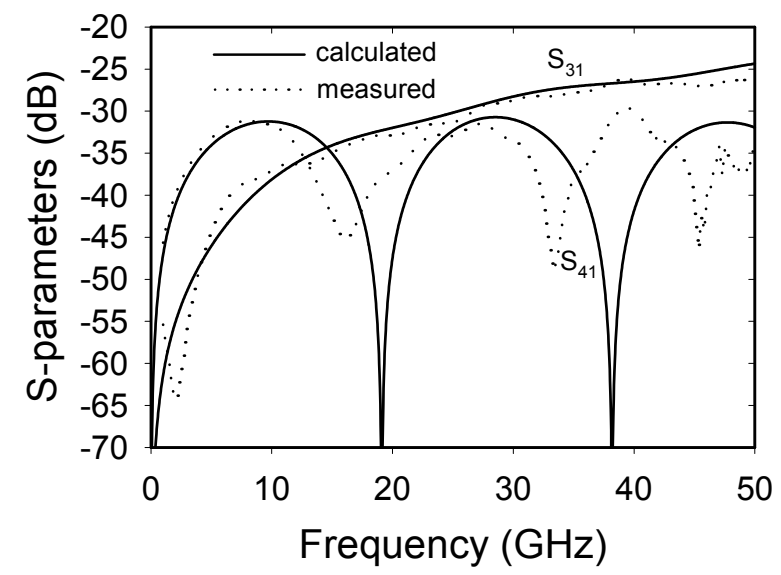

Figure 3: Comparison of measured and FDTD scattering parameters for coupled microstrip lines on the same polyimide layer (Figure 1a) with no shielding structure and $C=69 \mu \mathrm{m}$.

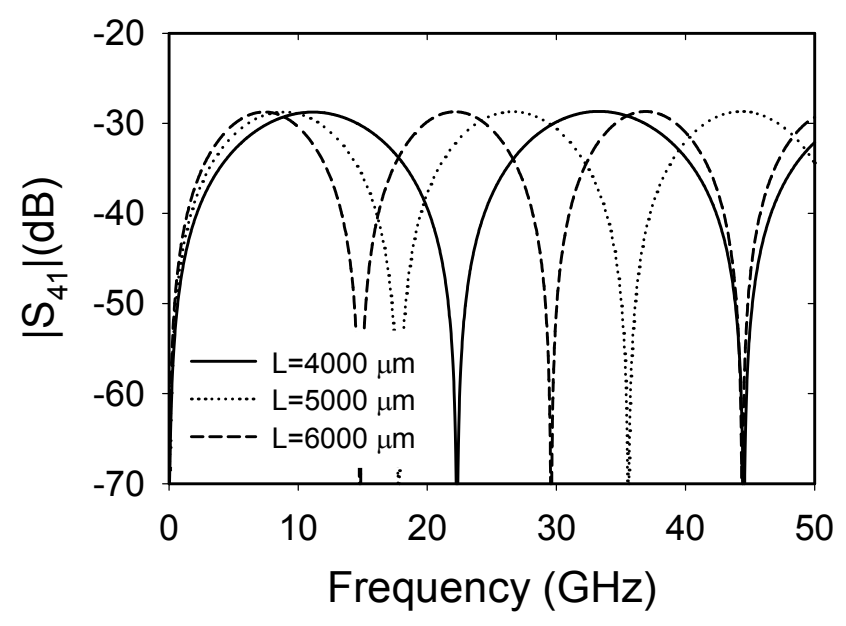

(a) 


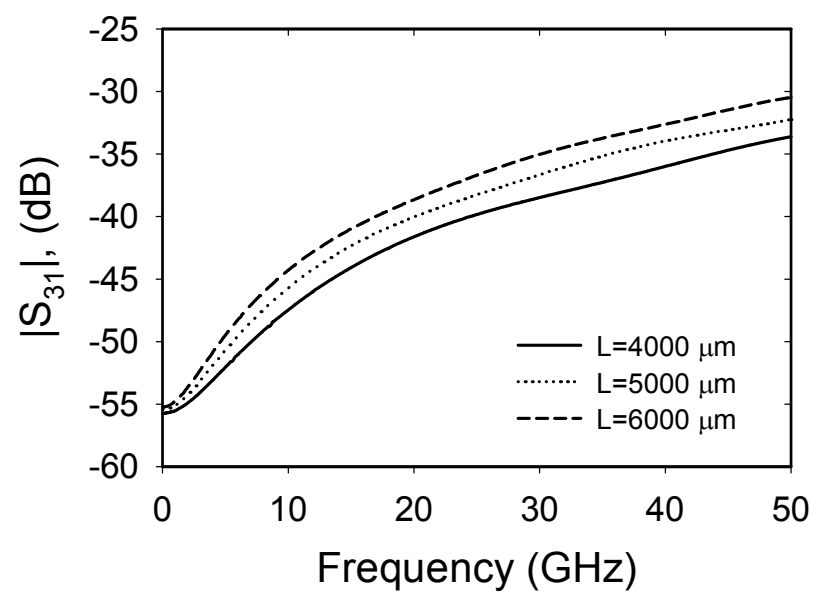

(b)

Figure 4: FDTD derived S-parameters as a function of coupling length and frequency for coupled microstrip lines on the same polyimide layer (Figure 1a) with a trench shielding structure and $\mathrm{C}=72 \mu \mathrm{m}$.

The effect of shielding structures on the coupling level between parallel microstrip lines fabricated on the same layer, as shown in Figure 1a, is summarized in Figure 5. For closely spaced microstrip lines without shielding structures, the backward coupling is slightly stronger than the forward coupling, but for widely spaced lines, the forward coupling is approximately $5 \mathrm{~dB}$ stronger. It is also seen that for closely spaced lines without any via hole structures, the coupling is very high (approximately $30 \mathrm{~dB}$ ), but the coupling decreases monotonically to approximately $45 \mathrm{~dB}$ as $\mathrm{C}$ increases.

When a shielding structure is introduced in layer 1 only (shield height equal to the microstrip height, $h$ ), the backward coupling is reduced by $8 \mathrm{~dB}$ for closely spaced lines, but there is very little improvement for larger line spacing or in the forward coupling. However, if the via fence or a continuous trench is placed through both layers of polyimide, the backward coupling is reduced by approximately $18 \mathrm{~dB}$ and the forward coupling is reduced by approximately $12 \mathrm{~dB}$ at 25 $\mathrm{GHz}$ for closely spaced lines. Moreover, it is seen that the use of shielding structures enables microstrip transmission lines to be placed as close as $60 \mu \mathrm{m}$ while yielding the same coupling as lines with no shielding structures placed $130 \mu \mathrm{m}$ apart.

It is interesting that the via fence interconnected by a metal strip yields the same coupling level as the metal filled trench. Also, although not shown, it was found that the results are not dependent on the via hole spacing (DV= 60 or 100 $\mu \mathrm{m})$. However, these results are expected when the dimensions of the rectangular mesh created by the vias and metal 
strips that interconnect them is compared to a wavelength. Using the largest dimensions that were characterized, the mesh is 10 by $80 \mu \mathrm{m}$, which at $50 \mathrm{GHz}$ results in the mesh being 0.003 by $0.023 \lambda_{\mathrm{d}}$ where $\lambda_{\mathrm{d}}$ is the wavelength in the dielectric. Thus, the via fence appears electrically to be a solid wall. Another interesting result shown in Figure 5 is that the coupling between microstrip lines with shielding structures (2 layer and trench) is very low for small $\mathrm{C}$, it then increases as $\mathrm{C}$ increases, and finally reduces again as $\mathrm{C}$ increases further. The small $\mathrm{C}$ result is believed to be true, but it is not very useful in practice because the microstrip line is nearly touching the shielding structure for small $\mathrm{C}$ and the microstrip fields are greatly perturbed. In fact, placing the shielding structure too close to the microstrip line results in an asymmetric surface current on the microstrip, resulting in a higher conductor loss.

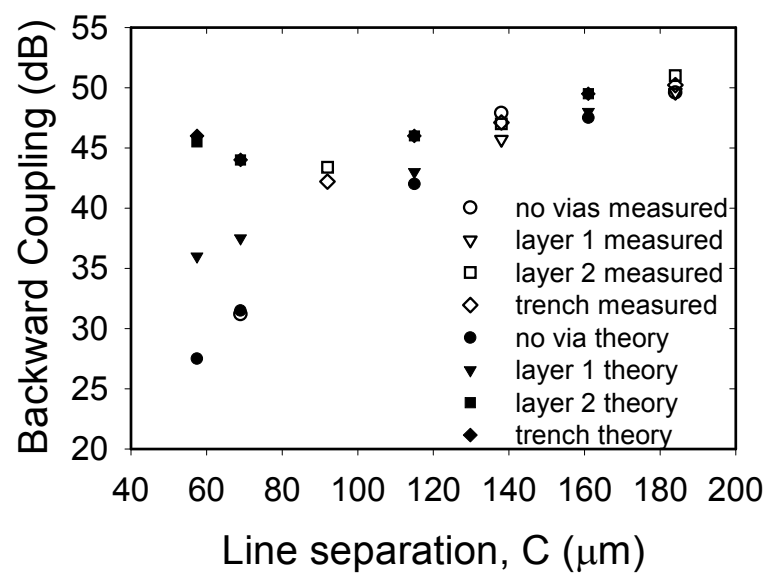

(a)

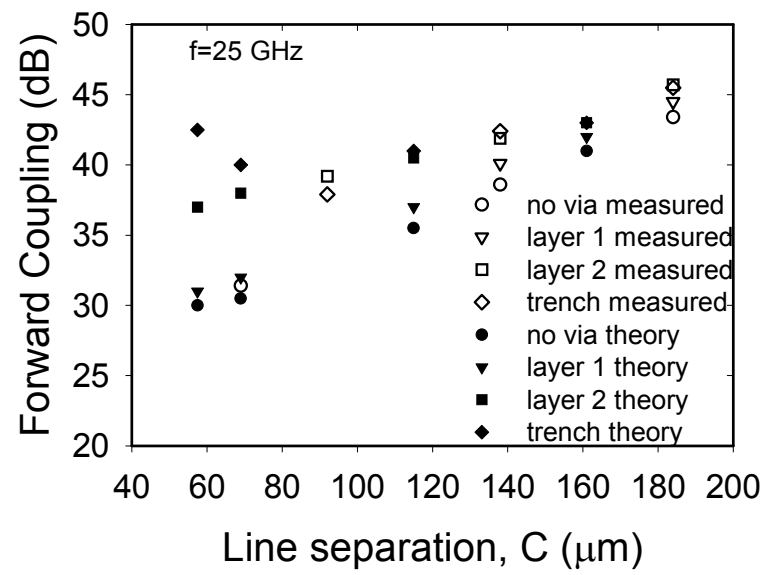

(b) 
Figure 5: Measured and FDTD (a) backward coupling and (b) forward coupling at 25 GHz of microstrip lines fabricated on the same layer of polyimide (Figure 1a) as a function of center to center spacing, $C$.

It is seen in Figure 6 that for all frequencies, coupling decreases as the line separation increases with the same slope. Furthermore, for any line separation, the shielding structure reduces forward coupling by the same amount across the entire frequency band. Lastly, as seen in Figure 3 and 6, forward coupling is very low at low frequencies, but increases rapidly with frequency. Therefore, at low frequencies, backward coupling dominates, but for frequencies greater than 25 $\mathrm{GHz}$, forward coupling dominates. Also, Figure 6 shows that the results shown in Figure $5 \mathrm{~b}$ and the conclusions drawn from them can be applied to the entire microwave frequency spectrum.

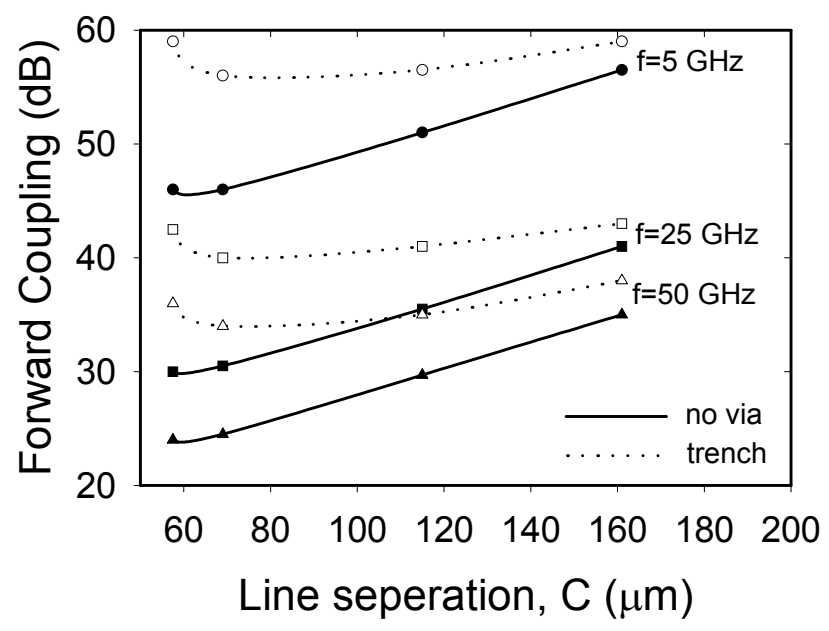

Figure 6: Forward coupling of microstrip lines fabricated on the same layer of polyimide (Figure 1a) as a function of frequency.

Coupling between unshielded microstrip lines is typically assumed to be dependent on the line separation, C, normalized by the substrate thickness, $\mathrm{H}$, or coupling is dependent on $\mathrm{C} / \mathrm{H}[12,13]$. To investigate the effect of shielding structures on this relationship, a set of coupled microstrip lines were fabricated and characterized as described previously, except $\mathrm{h}=5 \mu \mathrm{m}$ and $\mathrm{W} 1=12 \mu \mathrm{m}$. The via hole dimensions and trench were kept at the previously described dimensions. This yields a $50 \Omega$ microstrip line that is an exact scaled version of the previously reported results. Figure 7 shows the measured and FDTD determined backward and forward coupling for both sets of circuits ( $\mathrm{h}=10$ and $5 \mu \mathrm{m})$. It is seen that the results when there are no via holes (filled symbols) supports the assumption that coupling is dependent on $\mathrm{C} / \mathrm{h}$. Furthermore, the results also show that this relationship holds when shielding structures are used to minimize coupling. 
The measured coupling between microstrip lines fabricated on different layers of polyimide as shown in Figure $1 \mathrm{~b}$ are summarized in Figure 8. Generally, the observations pertaining to Figure 5 are true here as well, but the reduction in coupling with shielding structures is very small, approximately $5 \mathrm{~dB}$, which is the approximate reduction in coupling when the shielding structure height is equal to the substrate height, $\mathrm{h}$, in the previous results. Comparing the two different cases shown in Figures 1a and 1b, it is seen that microstrip lines embedded on the same layer of polyimide with shielding structures that extend to the top of polyimide have approximately $10 \mathrm{~dB}$ less coupling than microstrip lines on different layers with the same shielding structure. Thus, while it may be necessary to place microstrip transmission lines on different layers of polyimide to reduce circuit size, this will result in higher coupling. It has also been shown that these results scale with the polyimide thickness, which enables designers to use these results for all $50 \Omega$ microstrip lines embedded in polyimide.

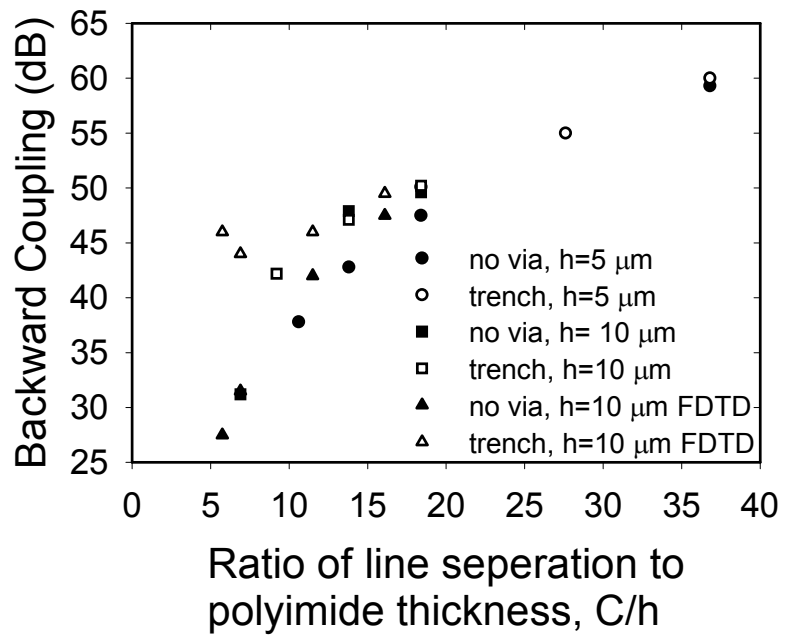

(a) 


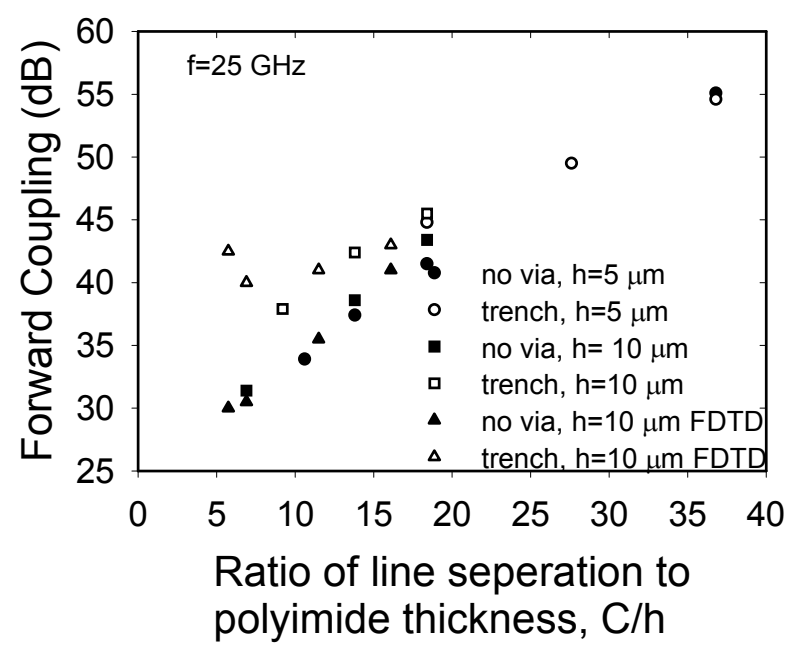

(b)

Figure 7: Backward and forward coupling between microstrip lines as shown in Figure 1a with $\mathrm{h}=10 \mu \mathrm{m}$ and $\mathrm{h}=\mathbf{5} \mu \mathrm{m}$. All lines are $50 \Omega$.

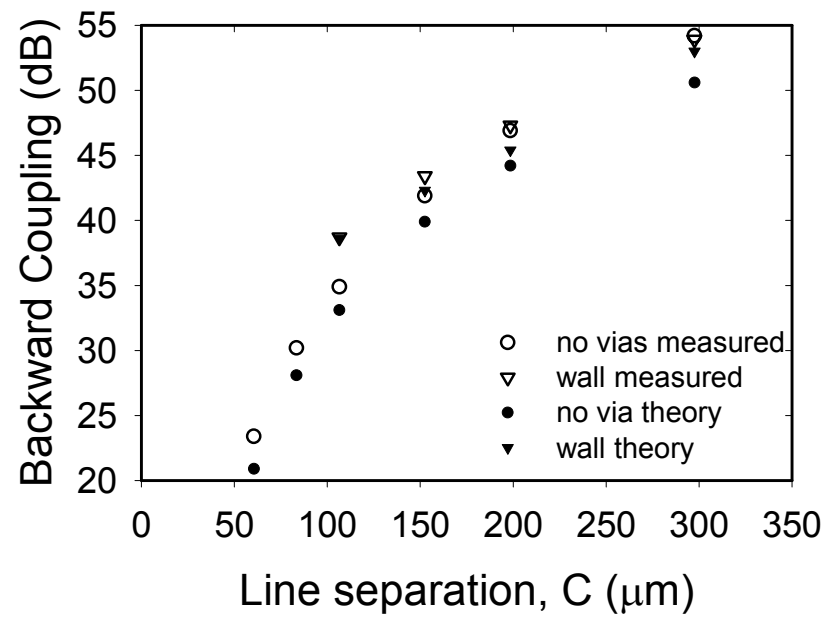

(a) 


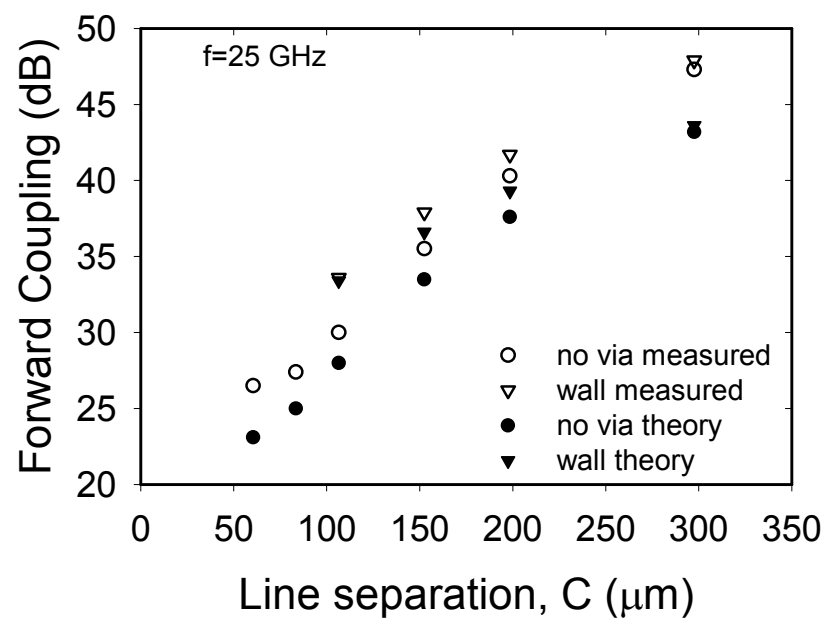

(b)

Figure 8: Measured (a) backward coupling and (b) forward coupling at $25 \mathrm{GHz}$ of microstrip lines fabricated on different layers of polyimide (Figure 1b) as a function of center-to-center spacing, $C$.

\section{Physics of Coupling Between Microstrip Lines}

Up to this point, it has been shown that shielding structures placed between adjacent microstrip lines can reduce the coupling between them, but the nature of the coupling and how the shielding structures work has not been explored. The FDTD analysis is useful for this discussion since it can generate electric and magnetic field plots, which provide insightful information.

Figure 9 shows the electric and magnetic field plots of two microstrip lines on the same layer of polyimide (Figure 1a) with no shielding structure. The line on the left is the excited line while the line on the right is the coupled line. Comparing the electric (Figure 9a) and magnetic (Figure 9b) field distributions, it is seen that the coupling is primarily due to the magnetic field. In fact, the maximum field magnitude under the center of the coupled line is $-34.28 \mathrm{~dB}$ for the electric field and $-32.83 \mathrm{~dB}$ for the magnetic field. Figure 10 shows the electric and magnetic field distributions for the same microstrip lines when a shielding structure extends to the top of the polyimide. Examining the electric field distribution in Figure 10a first, it is seen that the shield is very effective and reduces the maximum electric field under the center of the coupled line to $-45.72 \mathrm{~dB}$. However, the magnetic fields shown in Figure $10 \mathrm{~b}$ are able to encircle the coupled microstrip line and still excite the microstrip mode. The maximum magnetic field under the center of the coupled 
line is now $-41.57 \mathrm{~dB}$. Thus, with the shielding structure, the magnetic field coupling is more dominant than the electric field coupling compared to the case before adding the shielding wall.

Figures 11 and 12 show similar field plots for coupled microstrip lines on different layers of polyimide without and with shielding structures, respectively. It is again seen that shielding structures reduce the electric field more than the magnetic field; a $7.3 \mathrm{~dB}$ reduction in electric field compared to a $5.1 \mathrm{~dB}$ reduction in magnetic field. Thus, while shielding structures can be very effective at reducing coupling between microstrip lines embedded in polyimide, this is primarily due to a strong reduction in the electric field coupling and a moderate reduction in the magnetic field coupling.

To derive general conclusions about the effectiveness of shielding structures in reducing electric and magnetic fields, the FDTD analysis was used to determine the excited fields at different points without and with a shielding structure. The analysis was first completed as a function of frequency, but it is found that the ratio of fields shielded to unshielded is not frequency dependent, which confirms the conclusions drawn from Figure 6. Figure 13a shows the schematic of the single microstrip line that was analyzed without and with the shielding structure, which was a trench, and Figure 13b shows the ratio of shielded to unshielded electric and magnetic fields as a function of position. It is seen that for small horizontal distance, $\Delta$, the electric field is reduced more than the magnetic field, but for large $\Delta$, the magnetic field is reduced slightly more than the electric field. It is also seen that the field reduction is greatest close to the ground plane, and the field reduction decreases as the surface of the polyimide is approached. This confirms the coupling results of Figures 5 and 8 for coupled microstrip lines at 10 and $20 \mu \mathrm{m}$ respectively.

Lastly, it may be asked if the coupling between microstrip lines is due to electromagnetic fields that leak through the via fence or is coupling a result of electromagnetic fields that extend over the top of the wall. Figures 10 and 12 appear to indicate that the fields extend over the top of the wall, it was already stated that the mesh created by the via fence is electrically small and therefore appears as a continuous wall, and that the measured coupling for the via fence and a trench wall are the same. These all indicate that coupling is not due to leakage through the wall. To confirm this, a FDTD analysis was completed for a structure similar to that shown in Figure 13a, but in this case, the via fence was made to extend to a height of $100 \mu \mathrm{m}$, or ten time the microstrip substrate height. The level of the electromagnetic fields on the other side of the shield from the microstrip are determined to be at least $76 \mathrm{~dB}$ below the fields under the microstrip line. Since this is close to the numerical error of $-85 \mathrm{~dB}$, it is concluded that via fence shields interconnected by metal strips prevent electromagnetic field leakage, and coupling is due to fields that extend over the top of the shield. 


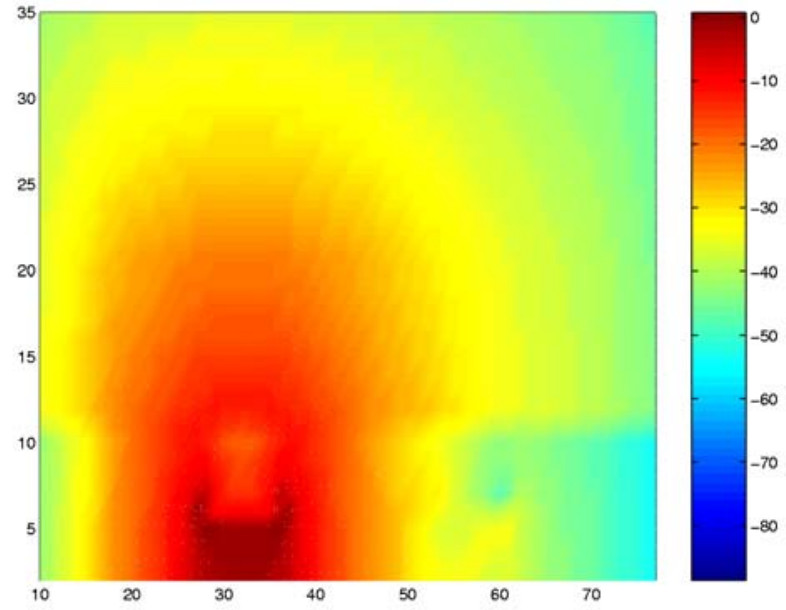

(a)

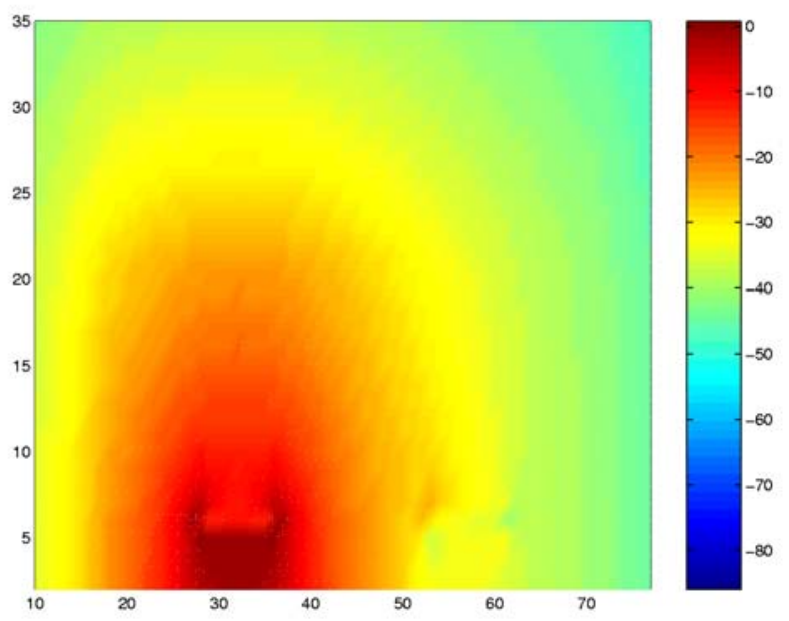

(b)

Figure 9: (a) Electric field and (b) magnetic field distribution for microstrip lines $(C=69 \mu \mathrm{m})$ on the same polyimide layer without shielding structure (Like Figure 1a). 


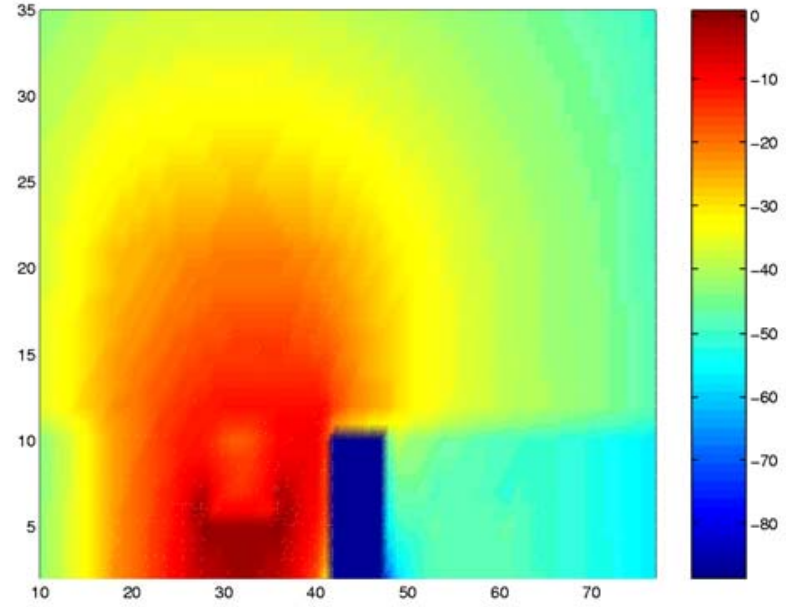

(a)

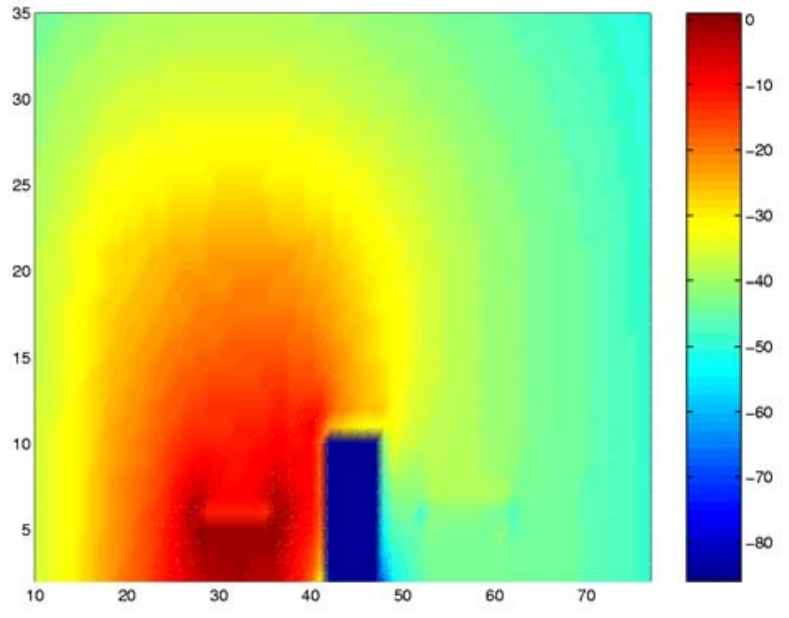

(b)

Figure 10: (a) Electric field and (b) magnetic field distribution for microstrip lines $(C=69 \mu \mathrm{m})$ on the same polyimide layer with shielding structure. 


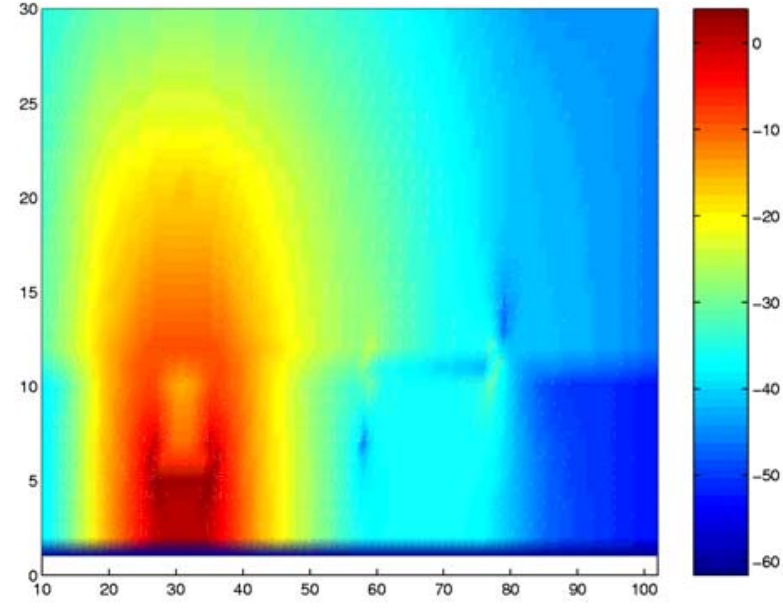

(a)

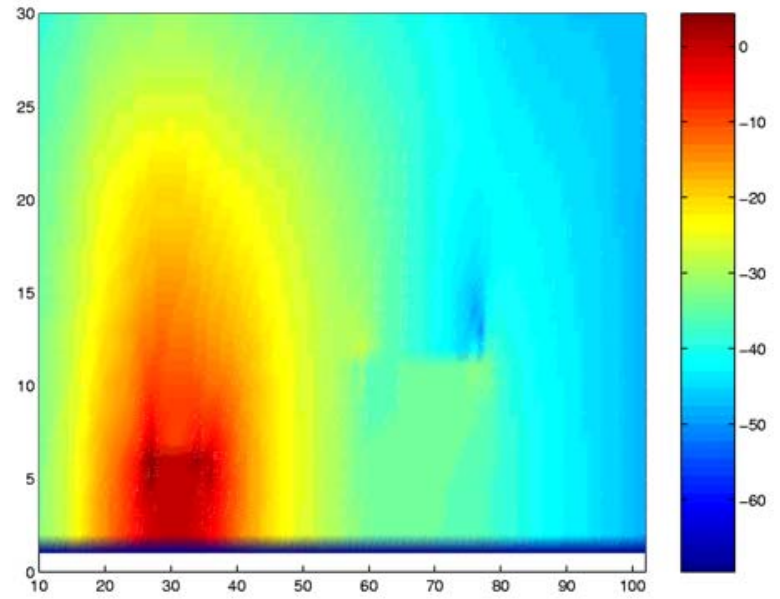

(b)

Figure 11: (a) Electric field and (b) magnetic field distribution for microstrip lines $(\mathrm{C}=106.5 \mu \mathrm{m})$ on different polyimide layers without shielding structure. 


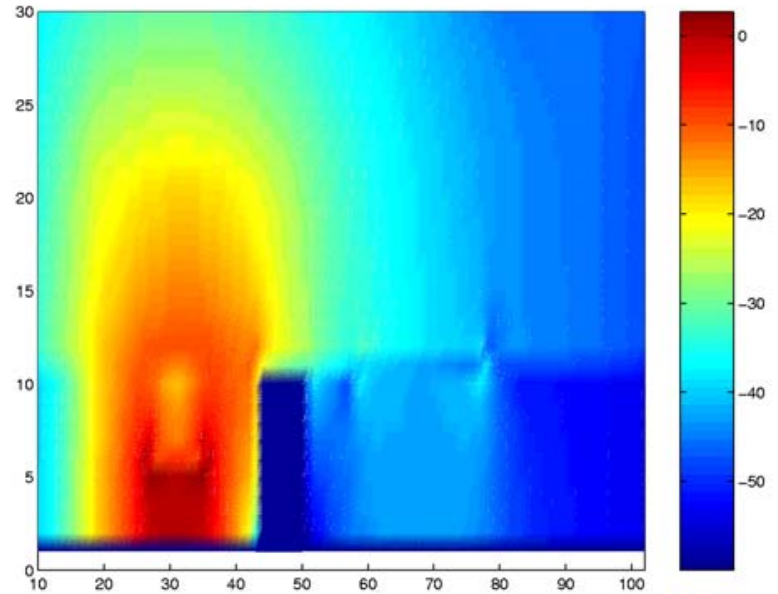

(a)

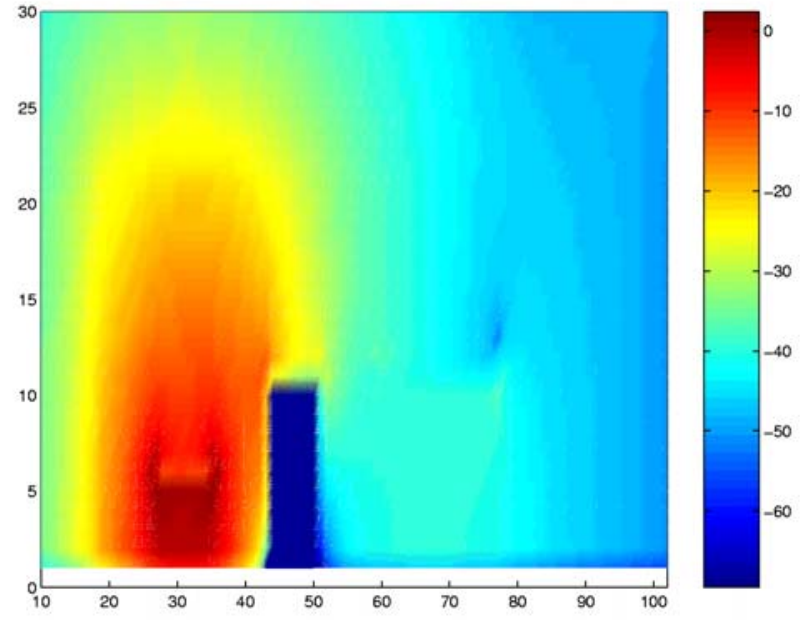

(b)

Figure 12: (a) Electric field and (b) magnetic field distribution for microstrip lines $(\mathrm{C}=106.5 \mu \mathrm{m})$ on different polyimide layers with shielding structure.

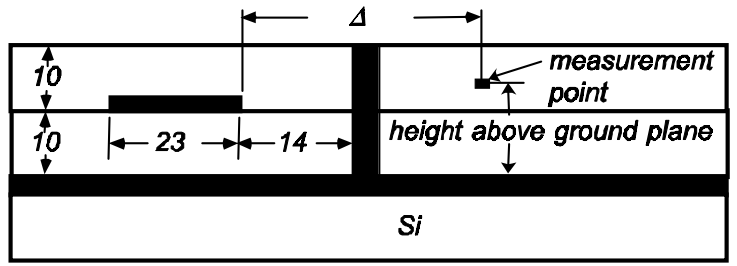

(a) 


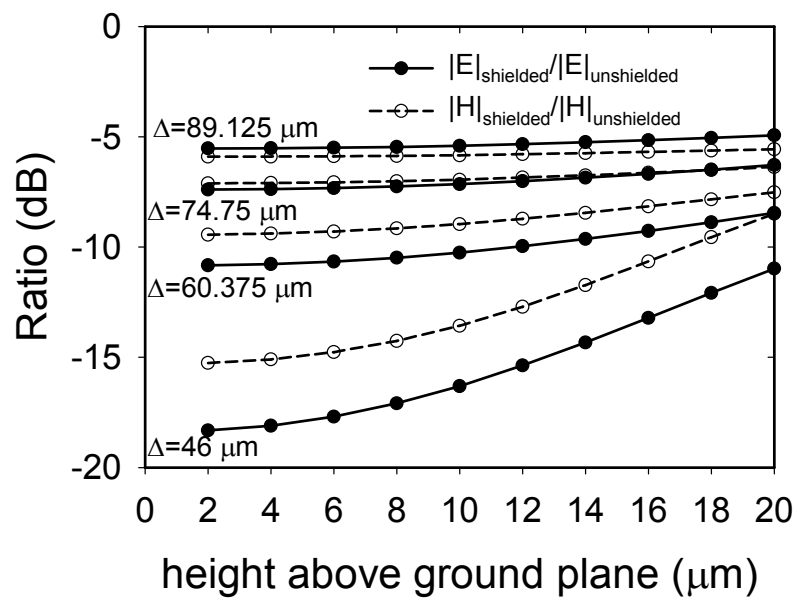

(b)

Figure 13: (a) Schematic of FDTD analyzed structure, and (b) reduction in electric and magnetic field due to a shielding structure as a function of position.

\section{CONCLUSION}

In this paper, a systematic evaluation of shielding structures for reducing coupling between microstrip lines embedded in polyimide has been presented. The results show that coupling is lower when both microstrip lines are on the same layer of polyimide and when the shielding structure extends through both layers of polyimide, or the shielding structures are more effective when they are higher than the microstrip substrate height. A shield comprised of metal posts interconnected by metal strips on the top and immediate layers provides the same shielding level as a continuous metal wall, at least to the measurement and numerical errors. The shielding structures are effective across the microwave spectrum, and it was shown that the results presented scale so that they are applicable to thinner or thicker polyimide circuits. Finally, it was shown that coupling between microstrip lines is primarily due to magnetic fields, and the shielding structures are effective in reducing both electric and magnetic field coupling.

\section{REFERENCES}

[1] G. E. Ponchak, "RF transmission lines on silicon substrates," 29 th European Microwave Conference Dig., Munich, Germany, Oct. 5-7, 1999, pp. 158-161. 
[2] G. E. Ponchak, D. Chun, J.-G. Yook, and L. P. B. Katehi, "The use of metal filled via holes for improving isolation in LTCC RF and wireless multichip packages," IEEE Trans. on Advanced Packaging, Vol. 23, No. 1, pp. 88-99, Feb. 2000.

[3] G. E. Ponchak, D. Chun, J.-G. Yook, and L. P. B. Katehi, "Experimental verification of the use of metal filled via hole fences for crosstalk control of microstrip lines in LTCC packages," IEEE Trans. on Advanced Packaging, Vol. 24, No. 1, pp. 76-80, Feb. 2001.

[4] K. Onodera, M. Hirano, M. Tokumitsu, I. Toyoda, K. Nishikawa, and T. Tokumitsu, "Folded U-shaped microwave technology for ultra-compact three-dimensional MMIC's," IEEE Trans. Microwave Theory Tech., Vol. 44, No. 12, pp. 2347-2353, Dec. 1996.

[5] G. E. Ponchak, E. M. Tentzeris, and J. Papapolymerou, "Coupling between microstrip lines embedded in polyimide layers for 3D-MMICs on Si," 2001 IEEE MTT-S Int. Microwave Symposium Dig., Phoenix, AZ, May 20-25, 2001, pp. 1723-1726.

[6] A. Taflove, Computational Electrodynamics, Artech House, Dedham, MA, 1995.

[7] L.Roselli, E.Tentzeris and L.P.B.Katehi, "Nonilnear circuit characterization using a multiresolution time domain technique," Proc. of the 1998 MTT-S Conference, pp.1387-1390, Baltimore, MD.

[8] J. Leu, H.-M. Ho, J. K. Lee, J. Kasthurirangan, C. N. Liao, and P. S. Ho, "The evaluation of low dielectric constant materials for deep submicron interconnect applications," in Proc. $6^{\text {th }}$ Meeting Dupont Symp. Polyimide Microelectronics, May 1-3, 1995.

[9] Dupont Company Pyralin LX data sheet.

[10] R. B. Marks, “A multilane method of network analyzer calibration,” IEEE Trans. Microwave Theory Tech., Vol. 39, pp. 1205-1215, July 1991.

[11]S. A. Schelkunoff and T. M. Odarenko, "Crosstalk between coaxial transmission lines," Bell System Technical Journal, Vol. 16, pp. 144-164, 1937.

[12]S. Kal, D. Bhattacharya, and N. B. Chakraborti, "Empirical relations for capacitive and inductive coupling coefficients of coupled microstrip lines," IEEE Trans. Microwave Theory Tech., Vol. MTT-29, No. 4, pp. 386-388, 1981. 
[13] A. J. Rainal, "Impedance and crosstalk of stripline and microstrip transmission lines," IEEE Trans. Components, Packaging, and Manufacturing Tech.- Part B, Vol. 20, No. 3, pp. 217-224, 1997. 